\title{
Progresses of Functional Magnetic Resonance Imaging Diagnosis in Breast Cancer
}

\author{
Qianfei Hu1, Sibin Liu \\ ${ }^{1}$ Department of Medicine, Yangtze University, Jingzhou, China \\ ${ }^{2}$ Radiology Department, Jingzhou Central Hospital, Jingzhou, China \\ Email:1157880518@qq.com
}

How to cite this paper: $\mathrm{Hu}, \mathrm{Q} . \mathrm{F}$. and $\mathrm{Liu}$, S.B. (2020) Progresses of Functional Magnetic Resonance Imaging Diagnosis in Breast Cancer. Yangtze Medicine, 4, 85-96. https://doi.org/10.4236/ym.2020.42009

Received: August 26, 2019

Accepted: June 26, 2020

Published: June 29, 2020

Copyright (c) 2020 by author(s) and Scientific Research Publishing Inc. This work is licensed under the Creative Commons Attribution International License (CC BY 4.0).

http://creativecommons.org/licenses/by/4.0/

\begin{abstract}
Breast cancer is the most common malignant tumor that threatens women's health. Breast magnetic resonance imaging (MRI) is a commonly used method recommended for the diagnosis of breast cancer. Diffusion weighted imaging (DWI) and dynamic enhanced magnetic resonance imaging (DCE-MRI) are now widely used. At present, with the continuous advancement of magnetic resonance technology, Magnetic resonance spectroscopy (MRS), Perfusion weighted imaging (PWI), Positron emission tomography-magnetic resonance imaging (PET-MRI) and so on are gradually being used in clinical practice. Mammography imaging and imaging genomics are hot topics. This article will briefly introduce several functional magnetic resonance techniques and their latest applications.
\end{abstract}

\section{Keywords}

Breast Cancer, Functional Magnetic Resonance Techniques, Diagnostic Imaging

\section{Introduction}

Breast cancer is the most common malignant tumor that threatens women's health. Early detection and accurate diagnosis are especially important. The American Academy of Radiology (ACR) recommended guidelines for the use of breast magnetic resonance [1], suggesting that magnetic resonance can be used not only for people with malignant lymph node metastasis and chest wall infiltration that cannot be accurately analyzed by other tests, but also for screening high-risk groups. Compared with other examinations, such as X-ray, CT and ultrasonography, in addition to clearly show the shape, location, size and signal characteristics of the lesion, breast MRI can also make a correct judgment of 
breast lesions according to the hemodynamic characteristics of the lesion, which is beneficial for early diagnosis of breast cancer [2]. At present, especially the development of magnetic resonance spectroscopy (MRS), dynamic enhanced magnetic resonance imaging (DCE-MRI) and diffusion-weighted imaging (DWI) have enabled breast cancer to enter the field of morphological and functional diagnosis [3], this article will briefly introduce several functional magnetic resonance techniques and their latest applications.

\section{Diffusion Weighted Imaging (DWI)}

DWI is a non-invasive method for detecting the dispersion of water molecules in living tissue at the level of molecular motion. It has the advantages of no radiation, no contrast agent, no invasiveness and high sensitivity. Meta-analysis showed that the sensitivity and specificity of differential diagnosis of breast cancer by DWI were $86.0 \%$ and 75.6\%, respectively [4] (as we can see in Figure 1). We usually use the apparent diffusion coefficient (ADC value) to reflect the dispersion characteristics. Studies [5] have shown that due to the rich blood supply, the ADC value of breast cancer is lower than that of benign lesions. The optimal threshold range for identifying the benign and malignant breast tumors ranged from $1.06 \times 10^{-3} \mathrm{~mm}^{2} / \mathrm{s}$ to $1.10 \times 10^{-3} \mathrm{~mm}^{2} / \mathrm{s}$ [6] [7]. Wang Shouhong et al. [8] analyzed 60 lymph node lesions in 40 patients with breast cancer, and finally found that the sensitivity, accuracy and specificity of DWI in evaluating lymph node metastasis of breast cancer reached $91.9 \%, 87.0 \%$ and $88.7 \%$, respectively. This suggests that DWI is of great value in assessing lymph node metastasis in breast cancer. In addition, Luo Ningbin [9] and others also demonstrated that $\mathrm{ADC}$ values can also reflect the proliferative activity of breast cancer cells, and help to evaluate the efficacy of neoadjuvant chemotherapy. At the same time, the sensitivity coefficient (b value) can also describe the diffusion ability of water molecules. It is very important to select the appropriate b value. Generation

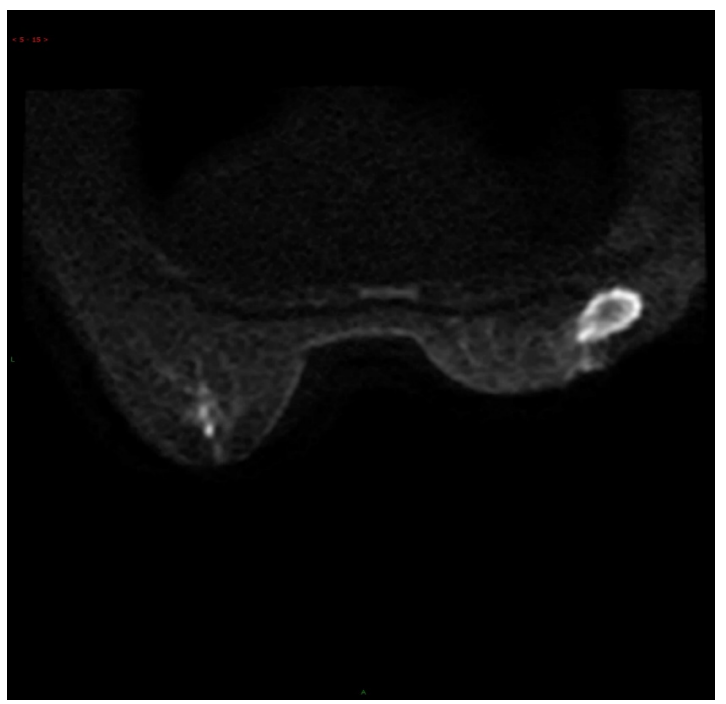

Figure 1. DWI image of breast cancer. The arrow refers to breast cancer lesions. 
Ocean et al. [10] calculated the ADC values of breast tumors under different $\mathrm{b}$-values, and found that when $\mathrm{b}$ value $=1000 \mathrm{~s} / \mathrm{mm}^{2}$, DWI has the highest sensitivity and specificity for the diagnosis of benign and malignant breast tumors. However, due to the poor signal-to-noise ratio of the DWI image, it is easy to be deformed, often causing missed diagnosis of small lesions. Therefore, it is necessary to combine other sequences in the diagnosis.

DWI includes a single exponential model, a double exponential model, and a tensile exponential model. The traditional DWI calculates the dispersion motion of water molecules in the interstitial space by a single exponential function. Intravoxel incoherent motion (IVIM) imaging is a double-exponential model, and IVIM is more specific for the diagnosis of breast cancer than DWI [11]. Another study [12] found that the specificity and accuracy of IVIM combined with DCE-MRI in the diagnosis of breast cancer were higher than DCE-MRI alone. The relevant parameters of the tensile index model include the diffusion coefficient (DDC) and the diffusion heterogeneity index $(\alpha)$. Compared with traditional DWI, DDC10\% combined with $\alpha$ mean to diagnose breast cancer has higher sensitivity and specificity [13]. At present, with the deep research of various index models of DWI, more precise individualized treatment is provided for breast cancer patients with different molecular subtypes (Table 1).

\section{Dynamic Enhanced Magnetic Resonance Imaging (DCE-MRI)}

Dynamic enhanced magnetic resonance imaging is a non-invasive imaging technique for evaluating angiogenesis of breast tumors and is recognized as the most sensitive imaging method for diagnosing breast cancer. Pinker et al. [14] found that DCE-MRI is $100 \%$ sensitive to breast cancer diagnosis, and the accuracy and specificity are as high as $96.6 \%$. And $90 \%$ (as we can see in Figure 2). In addition, compared with DCE-MRI or DWI, the two imaging methods combined with the diagnosis of breast cancer specificity (94\%), sensitivity (98\%) and accuracy (96\%) were high [15]. In addition to its high imaging sensitivity, DCE-MRI clearly shows the location, shape, size, and blood flow of the breast mass, by plotting the TIC (practice-signal intensity) curve of the breast mass for breast tumors. The diagnosis of benign and malignant provides a reference value (as we can see in Figure 3). The TIC curve is actually a semi-quantitative analysis method, so many scholars try to apply the DCE-MRI image information more objectively and quantitatively, and thus apply it to the field of breast diseases. DCE-MRI quantitative examination can reflect the blood perfusion and blood

Table 1. Comparison of different models of DWI.

\begin{tabular}{ccc}
\hline Different models of DWI & Sensitivity & Specificity \\
\hline Single exponential model & $86.0 \%$ & $75.6 \%[4]$ \\
Double exponential model & $90.5 \%-93.1 \%$ & $94.8 \%-95.7 \%[11]$ \\
Tensile exponential model & $90.2 \%$ & $95.5 \%[13]$ \\
\hline
\end{tabular}




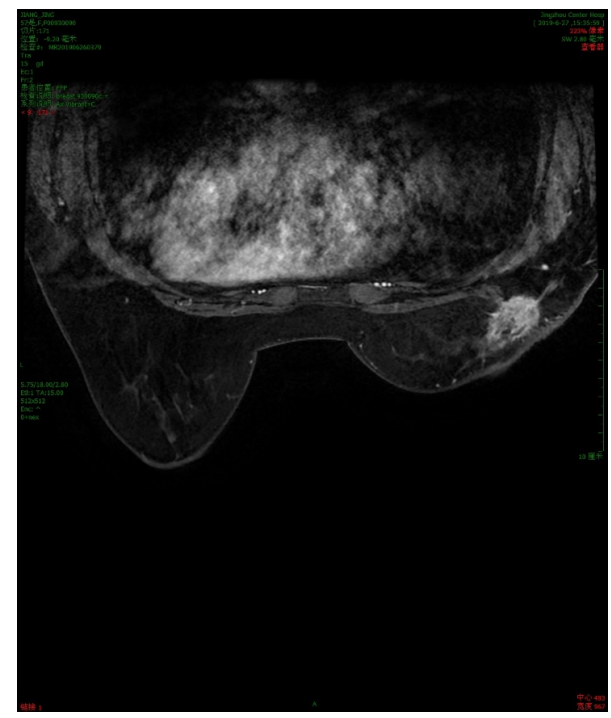

Figure 2. Dynamic enhanced magnetic resonance imaging of breast cancer, showing obvious enhancement of lesions.

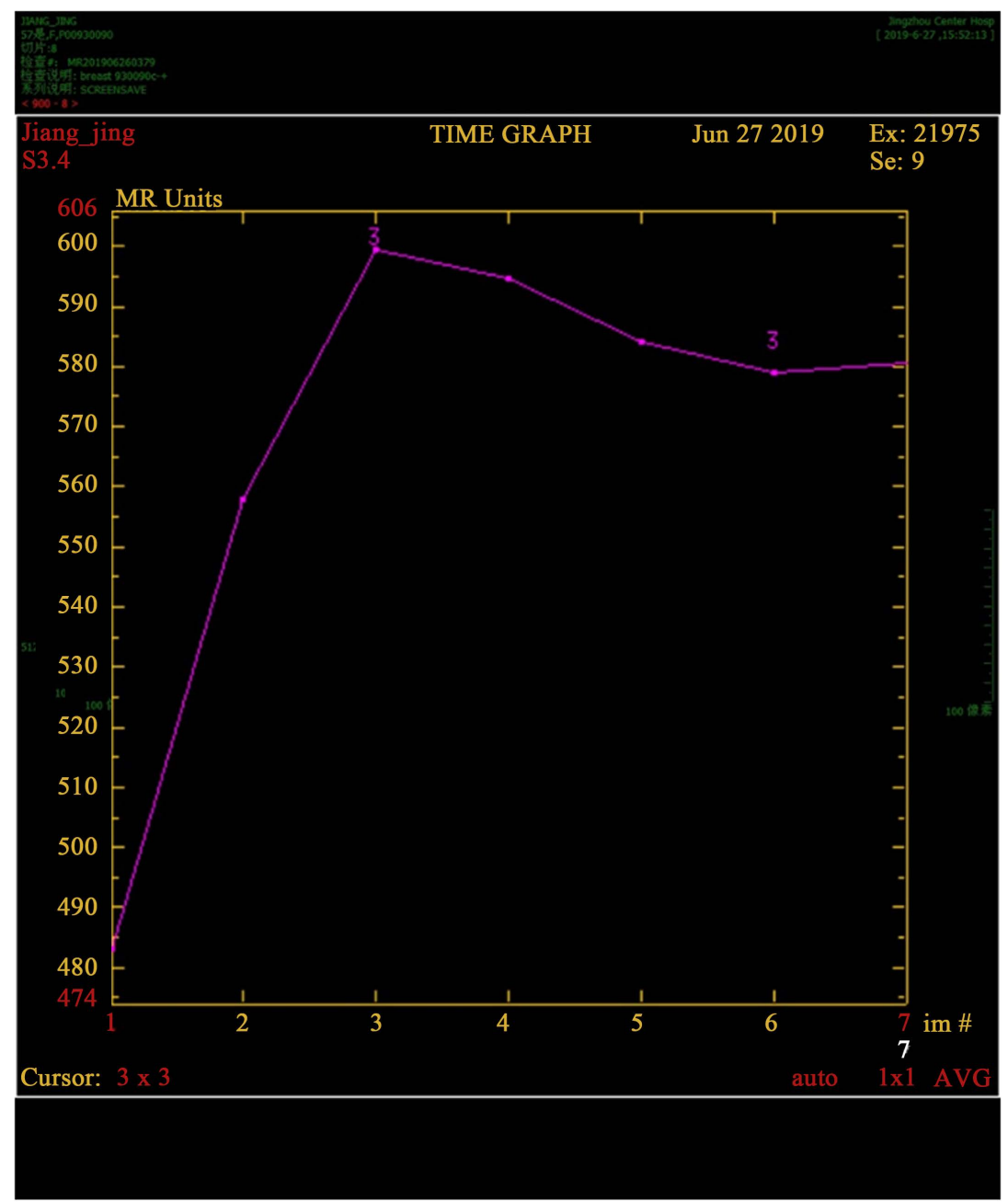

Figure 3. TIC curve of breast cancer lesions. This image curve represents the way in which breast cancer is strengthened. 
vessel distribution of tumor tissues at the molecular level, and make quantitative judgment on the nature of breast tumors [16]. The study [17] showed that Ktrans and Kep are feasible for the diagnosis of benign and malignant breast diseases, and the complete quantification of parameters can show the difference of angiogenesis in benign and malignant tumors. The diagnosis of benign and malignant breast tumors is more intuitive and accurate.

In addition, DCE-MRI is also used in the histological grade, prognosis assessment and molecular typing of breast cancer. Hye Ryoung Koo et al. [18] have shown that tumors with relatively low Ktrans and Kep values have relatively few neovascularizations, tumor vascular maturation is higher, tumor malignancy is lower, and prognosis is better. Shin et al. [19] found that using Ktrans values combined with ADC values can help identify low-risk breast cancer and is instructive for treatment decisions. The results of the study [20] showed that the smaller the Ktrans and Kep values, the lower the Ki-67 degree, and the greater the possibility that the ER and PR were positive, indicating that the breast tumor is highly differentiated, low malignant, and the better the prognosis.

In conclusion, the quantitative parameters of DCE-MRI not only have predictive value for the prognosis of patients with different subtypes of breast cancer, but also help to select individualized treatment options.

\section{Magnetic Resonance Spectroscopy (MRS)}

Magnetic resonance spectroscopy (MRS) is a promising non-invasive diagnostic technique for studying breast cancer metabolism. MRS can analyze changes in the content of choline and its related metabolites in the body, thereby showing the metabolic and biochemical information of the lesions in the human body to diagnose the disease. We can identify breast tumors by observing changes in the choline concentration of the breast tissue. Yeung [21] found that the sensitivity of MRS in the diagnosis of breast cancer was $92 \%$, the specificity was $83 \%$, and the accuracy was $90 \%$. At the same time, in addition to the analysis of choline peaks, Thakur et al. [22] found that quantitative assessment of lipid metabolism in vivo can help distinguish breast benign and malignant tumors, distinguish breast cancer molecular subtypes, and predict long-term survival outcomes, thus providing a possible Additional non-invasive imaging biomarkers to guide the decision to treat breast cancer. Galati [23] and other 132 patients with breast cancer confirmed by biopsy performed 3T breast MR imaging. Finally, it was found that there was a significant difference between the appearance of choline peak and the increase of tumor grade, Ki-67 value and lesion range. Correlation. This indicates that MRS is an effective means to further understand breast cancer biological information and predict tumor invasiveness.

In addition, according to the relevant study [24], the accuracy and specificity of MRS combined with DCE-MRI in the diagnosis of breast cancer were higher than that of MRS or DCE-MRI alone, indicating the combined use of MRS and DCE-MRI has better diagnostic performance in assessing suspected breast le- 
sions. However, because MRS imaging technology is affected by many factors, such as longer imaging time and difficulty in standardizing choline concentration, the application of MRS needs further improvement.

\section{Perfusion Weighted Imaging (PWI)}

Magnetic resonance perfusion imaging is an imaging technique that reflects the microvascular distribution and blood perfusion of breast tissue. There are significant differences in the number of blood vessels and the permeability of blood vessels between healthy people and breast cancer patients, so there is a significant difference in signal intensity during perfusion imaging. Because of the large specific range of magnetic resonance dynamic enhancement diagnosis, it is recommended in the literature [25] to use $\mathrm{T} 2{ }^{*} \mathrm{WI}$ to identify benign and malignant lesions of the breast. T2 ${ }^{*} \mathrm{WI}$ can better reflect the microvascular distribution of tumor tissue and the microcirculation of tumor tissue, especially the characteristics of microvascular permeability [26]. According to the study [27], the sensitivity of PWI for the diagnosis of breast cancer was $79 \%-90.3 \%$, the specificity was $83.8 \%-93 \%$, and the accuracy was $82.61 \%-85.0 \%$. Moreover, $\mathrm{Li}$ et al. [28] found that the sensitivity of T2* WI in the identification of benign and metastatic lymph nodes was $94.6 \%$ and the specificity was $98.5 \%$ by studying the axillary lymph nodes of 35 breast cancer women. This suggests that $\mathrm{T} 2$ * WI in vivo can help distinguish between benign and metastatic axillary lymph nodes in breast cancer patients, thus providing a basis for subsequent surgery/chemotherapy. In addition, in terms of neoadjuvant chemotherapy, Wang Xiaohong et al. [29] found that T2 * WI can also evaluate and predict the efficacy of neoadjuvant chemotherapy.

There are many shortcomings in the application of MR perfusion weighting in the diagnosis of breast cancer, For example, the signal intensity-time curve of the selected region of interest does not represent the perfusion of the entire tumor, because the tumor is in vivo. There is heterogeneity in blood perfusion. Moreover, $\mathrm{T} 2$ * WI has a higher diagnostic specificity for breast cancer, but its sensitivity is lower. Therefore, the combination of $\mathrm{T} 1$ dynamic enhancement and perfusion imaging can fully improve the diagnostic specificity (Table 2).

\section{PET/MRI}

PET/MRI is a molecular level functional imaging and structure imaging system

Table 2. Comparison of different magnetic resonance scanning methods in diagnosis of breast cancer.

\begin{tabular}{cccc}
\hline Imaging method & Sensitivity & Accuracy & Specificity \\
\hline DCE-MRI & $100 \%$ & $96.6 \%$ & $90.0 \%[14]$ \\
DCE-MRI combined with DWI & $98.0 \%$ & $96.0 \%$ & $94.0 \%[15]$ \\
MRS & $92.0 \%$ & $90.0 \%$ & $83.0 \%[21]$ \\
PWI & $79.0 \%-90.3 \%$ & $82.61 \%-85.0 \%$ & $83.8 \%-93.0 \%[27]$ \\
\hline
\end{tabular}


that combines PET (positron emission tomography) and MRI (magnetic resonance tomography) technology. The combination of the two can fully exert their respective advantages and have a higher accuracy in the diagnosis of breast diseases [30]. Some studies [31] found that the accuracy of PET/MRI combined with DCE-MRI in the diagnosis of breast cancer was $20 \%$ higher than that of DCE-MRI alone, and the specificity could be increased to 97\%. Xu Yuanfan [32] and others retrospectively analyzed 59 patients with suspected breast tumors, and let them perform PET/CT and PET/MRI examinations on the same day. Finally, the sensitivity, specificity and accuracy of PET/MR were higher than PET/CT. However, because PET/MRI instruments are relatively expensive, there are fewer technicians and diagnosticians with expertise in processing and diagnosing images of PET/MRI, thereby limiting its use.

\section{Mammography Imaging and Imaging Genomics}

Image grouping was proposed by Gillies [33] and further refined by scholars Lambin et al. [34], which refers to the acquisition of a large number of characteristic image parameters from high-throughput medical image data, thereby uncovering image features and diseases. Association. Image grouping is divided into five parts, namely image acquisition, segmentation of lesions, feature extraction of lesion images, database establishment and data analysis [35]. Studies [36] have shown that mammography can effectively identify the benign and malignant breast diseases and improve the consistency of MRI diagnosis and pathological diagnosis. Whitney et al. [37] found that morphological features extracted from DCE-MRI images were helpful in distinguishing Luminal A breast and benign breast lesions. Breast magnetic resonance imaging has developed rapidly in recent years, which not only helps to identify the benign and malignant breast diseases, but also has a wide range of applications in molecular classification of breast cancer, evaluation of prognostic factors and risk of recurrence. For example, study [38] found that HR+ tumor cells have higher entropy values than HR-cells. In addition, Hui et al. [39] retrospectively analyzed 84 breast cancer patients and found that the enhanced scan texture analysis parameter values were inversely proportional to the risk of recurrence.

Image genomics refers to the study of the relationship between genomic features and imaging features of breast cancer, and then uses image features to reflect gene activity and ultimately guide the diagnosis and prevention of breast cancer [40]. Mammography genomics is developing rapidly. With the advent of the era of big data, mammography and imaging genomics are the future development trend (Table 3 ).

\section{Summary and Outlook}

In summary, with the continuous advancement of magnetic resonance technology, the magnetic resonance diagnosis method of the breast is also constantly developing. Currently, the role of diffusion-weighted imaging and dynamic 
Table 3. Comparison of six magnetic resonance imaging methods. These six methods can be used to diagnose breast cancer, each with its own advantages and disadvantages. But the first two are the most widely used in clinical practice.

\begin{tabular}{|c|c|c|c|c|}
\hline Method & Definition principle & Advantage & Disadvantage & Clinical application \\
\hline DWI & $\begin{array}{l}\text { Detection of diffusion } \\
\text { of water molecules } \\
\text { in living tissue }\end{array}$ & $\begin{array}{l}\text { No contrast agent; } \\
\text { High sensitivity; } \\
\text { Easy to operate; }\end{array}$ & $\begin{array}{l}\text { Low image resolution; } \\
\text { Low specificity }\end{array}$ & $\begin{array}{l}\text { Most basic and } \\
\text { widely used }\end{array}$ \\
\hline DCE-MRI & $\begin{array}{l}\text { Evaluation of breast } \\
\text { tumor angiogenesis }\end{array}$ & $\begin{array}{c}\text { High sensitivity, accuracy } \\
\text { and specificity; } \\
\text { Quantitative judgment }\end{array}$ & Complex to operate; & $\begin{array}{l}\text { Most basic and } \\
\text { widely used }\end{array}$ \\
\hline MRS & $\begin{array}{l}\text { Study changes in tumor } \\
\text { metabolite content }\end{array}$ & $\begin{array}{l}\text { analyze metabolites; } \\
\text { Quantitative assessment } \\
\text { of lipid metabolism }\end{array}$ & $\begin{array}{l}\text { Longer imaging time; } \\
\text { Quantification of choline } \\
\text { concentration is difficult } \\
\text { to standardize }\end{array}$ & Frequently used \\
\hline PWI & $\begin{array}{l}\text { Reflecting microvascular } \\
\text { distribution and blood } \\
\text { perfusion status } \\
\text { of breast tissue }\end{array}$ & $\begin{array}{c}\text { Reflect microvascular } \\
\text { distribution and blood } \\
\text { perfusion status; } \\
\text { Higher diagnostic specificity }\end{array}$ & $\begin{array}{c}\text { Low sensitivity; } \\
\text { Heterogeneity in blood } \\
\text { perfusion; }\end{array}$ & Less used \\
\hline PET/MRI & $\begin{array}{l}\text { A system of functional } \\
\text { imaging and structural } \\
\text { imaging at the } \\
\text { molecular level }\end{array}$ & $\begin{array}{l}\text { Functional imaging at the } \\
\text { molecular level; } \\
\text { High sensitivity, } \\
\text { accuracy and specificity; }\end{array}$ & $\begin{array}{l}\text { Complex to operate; } \\
\text { Less technicians and } \\
\text { diagnosticians; } \\
\text { Radioactivity expensive }\end{array}$ & Rarely used \\
\hline $\begin{array}{l}\text { Mammography imaging } \\
\text { and imaging genomics }\end{array}$ & $\begin{array}{l}\text { Studying the link } \\
\text { between breast cancer } \\
\text { genomic features and } \\
\text { imaging features }\end{array}$ & $\begin{array}{l}\text { Create a } \\
\text { high-dimensional data set; } \\
\text { Using image features } \\
\text { to reflect gene activity }\end{array}$ & $\begin{array}{l}\text { Too small sample size; } \\
\text { Clinically practical is } \\
\text { not strong }\end{array}$ & $\begin{array}{c}\text { Used only for } \\
\text { academic research }\end{array}$ \\
\hline
\end{tabular}

enhanced magnetic resonance imaging in the diagnosis of breast cancer has been recognized. Imaging techniques such as MRS, PWI, and PET-MRI are limited in their use. With the advancement of magnetic resonance technology, many new imaging technologies have been born, such as blood oxygen level-dependent functional magnetic resonance imaging, elastic imaging technology, and breast magnetic sensitive imaging.

Advanced and cost-effective magnetic resonance equipment should promote the research and clinical application of breast imaging worldwide. Here we have summarized the magnetic resonance equipment from home and abroad (Table 4). At present, China's magnetic resonance equipment is mainly from imports, and magnetic resonance equipment of domestic brands (Shanghai United Imaging Healthcare, Neusoft, Alltech) is also available in China and exported to developed countries. Since some models of domestic MRI equipment combined with the actual situation of China's domestic medical requirement, the overall experience of domestic equipment is significantly higher than that of imported brands [41]. Moreover, the investment recovery period of domestic magnetic resonance equipment only needs 2.5 years [42], which has a good diagnostic cost performance. However, due to the late start of domestic equipment, MRI practitioners have a higher level of understanding of the imported brands than domestic brands, and the application of domestic brand machines requires more 
Table 4. Main domestic and imported magnetic resonance equipment and models.

\begin{tabular}{ccc}
\hline Field strength & Brand & Main model \\
\hline & Shanghai United Imaging Healthcare & uMR770 uMR780 \\
Siemens & MAGNETOM Verio \\
Philips & Achieva Ingenia \\
GE & HDxt \\
Neusoft & NSM-S15P \\
Alltech & Centauri Echo Star \\
& uMR 560 uMR570 \\
& Shanghai United Imaging Healthcare & MAGNETOM Avanto \\
& Siemens & Achieva Ingenia \\
& Philips & HDx \\
\hline
\end{tabular}

education of MRI practitioners [43]. Finally, I believe that with the advancement and development of medical technology, the future research and application progress of breast magnetic resonance can be expected.

\section{Conflicts of Interest}

The authors declare no conflicts of interest regarding the publication of this paper.

\section{References}

[1] Spak, D.A., Plaxco, J.S., Santiago, L., Dryden, M.J. and Dogan, B.E. (2017) BI-RADS ${ }^{\circledast}$ Fifth Edition: A Summary of Changes. Diagnostic and Interventional Imaging, 98, 179-190. https://doi.org/10.1016/j.diii.2017.01.001

[2] Zhang, L., Han, L.X., Cao, H.X., Du, Y.Q., Chen, W. and Wang, J. (2017) The Value of 3.0 T Magnetic Resonance Diffusion Weighted Imaging and VIBRANT Dynamic Enhancement in the Differential Diagnosis of Breast Adenosis and Breast Cancer. Journal of Clinical Radiology, 36, 342-346.

[3] Shao, G.L., Fan, L.Y., Zhang, J., Dai, G. and Xie, T.M. (2017) Association of DW/DCE-MRI Features with Prognostic Factors in Breast Cancer. The International Journal of Biological Markers, 32, e118-e125. https://doi.org/10.5301/jbm.5000230

[4] Zhang, L., Tang, M., Min, Z.Q., Lu, J., Lei, X.Y. and Zhang, X.L. (2016) Accuracy of Combined Dynamic Contrast-Enhanced Magnetic Resonance Imaging and Diffusion-Weighted Imaging for Breast Cancer Detection: A Meta-Analysis. Acta Radiologica (Stockholm, Sweden: 1987), 57, 651-660. https://doi.org/10.1177/0284185115597265

[5] McDonald, E.S., Hammersley, J.A., Chou Shinn-Huey, S., Habib, R., Scheel, J.R., Lee, C.I., Liu, C.-L., Lehman, C.D. and Partridge, S.C. (2016) Performance of DWI as a Rapid Unenhanced Technique for Detecting Mammographically Occult Breast Cancer in Elevated-Risk Women with Dense Breasts. American Journal of Roentgenology, 207, 205-216. https://doi.org/10.2214/AJR.15.15873

[6] Wan, C.W.S., Lee, C.Y., Lui, C.Y., Fong, C.Y. and Lau, K.C.H. (2016) Apparent 
Diffusion Coefficient in Differentiation between Malignant and Benign Breast Masses: Does Size Matter. Clinical Radiology, 71, 170-177. https://doi.org/10.1016/j.crad.2015.11.006

[7] Rabasco, P., Caivano, R., Simeon, V., Dinardo, G., Lotumolo, A., Gioioso, M., Villonio, A., Iannelli, G., D’Antuono, F., Zandolino, A., Macarini, L., Guglielmi, G. and Cammarota, A. (2017) Can Diffusion-Weighted Imaging and Related Apparent Diffusion Coefficient Be a Prognostic Value in Women with Breast Cancer. Cancer Investigation, 35, 92-99. https://doi.org/10.1080/07357907.2016.1267740

[8] Wang, S.H., Song, X.D., Zhao, Y.S., et al. (2018) Diagnostic Value of MR-DWI for Axillary Lymph Node Metastasis of Breast Cancer. Hebei Medical Journal, 40, 2127-2130.

[9] Luo, N.B., Suo, K., Huang, X.Y., Jin, G.Q., Liu, L.D. and Zhao, Y. (2018) Correlation between ADC Values of MR Diffusion-Weighted Imaging and Ki-67 Expression before and after Neoadjuvant Chemotherapy for Breast Cancer. Journal of Clinical Radiology, 37, 922-925.

[10] Dai, H.Y., Liu, G.R., Yan, S.Z., Huang, C.Y. and Chen, S.S. (2015) The Value of Different b-Value MR Diffusion-Weighted Imaging in Differential Diagnosis of Benign and Malignant Breast Tumors. Magnetic Resonance Imaging, 6, 829-832.

[11] Dijkstra, H., Dorrius, M.D., Wielema, M., et al. (2016) Senti-Automated Quantitative Intravoxel Incoherent Motion Analysis and Its Implementation in Breast Diffusion-Weighted Imaging. Journal of Magnetic Resonance Imaging, 43, 1122-1131. https://doi.org/10.1002/jmri.25086

[12] Ma, D.J., Lu, F., Zou, X.X., Zhang, H., Li, Y.Y., Zhang, L., Chen, L., Qin, D.J. and Wang, B. (2017) Intravoxel Incoherent Motion Diffusion-Weighted Imaging as an Adjunct to Dynamic Contrast-Enhanced MRI to Improve Accuracy of the Differential Diagnosis of Benign and Malignant Breast Lesions. Magnetic Resonance Imaging, 36, 175-179. https://doi.org/10.1016/j.mri.2016.10.005

[13] Liu, C.L., Wang, K., Li, X.D., Zhang, J.N., Ding J., Karl, S., Timothy, D., Liang, C.H. and Huang, C. (2017) Breast Lesion Characterization Using Whole-Lesion Histogram Analysis with Stretched-Exponential Diffusion Model. Journal of Magnetic Resonance Imaging, 47, 1701-1710. https://doi.org/10.1002/jmri.25904

[14] Pinker, K., Bogner, W., Baltzer, P., Trattnig, S., Gruber, S., Abeyakoon, O., Bernathova, M., Zaric, O., Dubsky, P., Bago-Horvath, Z., Weber, M., Leithner, D. and Helbich, T.H. (2013) Clinical Application of Bilateral High Temporal and Spatial Resolution Dynamic Contrast-Enhanced Magnetic Resonance Imaging of the Breast at 7 T. European Radiology, 24, 913-920. https://doi.org/10.1007/s00330-013-3075-8

[15] Wen, D.L. (2018) The Value of Dynamic Contrast-Enhanced Magnetic Resonance Imaging Combined with Magnetic Resonance Diffusion-Weighted Imaging in the Diagnosis of Breast Cancer Patients. Journal of Practical Medical Imaging, 19, 131-133.

[16] Liu, W.H. (2016) Progress and Research Direction of Quantitative MRI in Breast. Chinese Journal of Radiology, 50, 321-323.

[17] Li, R.M., Gu, Y.J., Mao, J., et al. (2011) Quantitative Dynamic Enhancement MRI for Differential Diagnosis of Benign and Malignant Breast Lesions. Chinese Journal of Radiology, 45, 164-169.

[18] Kim, W.H., Chang, J.M., Koo, H.R., et al. (2016) Impact of Prior Mammograms on Combined Reading of Digital Mammography and Digital Breast Tomosynthesis. Acta Radiologica, 58, 148-155. https://doi.org/10.1177/0284185116647211

[19] Shin, H.J., Kim, H.H., Shin, K.C., Sung, Y.S., Cha, J.H., Lee, J.W., Son, B.H. and 
Ahn, S.H. (2016) Prediction of Low-Risk Breast Cancer Using Perfusion Parameters and Apparent Diffusion Coefficient. Magnetic Resonance Imaging, 34, 67-74. https://doi.org/10.1016/j.mri.2015.10.028

[20] Fei, Y., Xu, J.M., Shen, Y.K., et al. (2016) Correlation between Quantitative Parameters of DCE-MRI and Biological Prognostic Factors in Breast Cancer. Journal of Medical Imaging, 26, 1625-1628.

[21] Yeung, D.K., Cheung, H.S. and Tse, G.M. (2001) Human Breast Lesions: Characterization with Contrast-Enhanced in Vivo Proton MR Spectroscopy-Initial Results. Radiology, 220, 40-46. https://doi.org/10.1148/radiology.220.1.r01j10240

[22] Thakur, S.B., Horvat, J.V., Ileana, H., Sutton, O.M., Bernard-Davila, B., Michael, W., Hun, O.J., Adele, M.M., Daly, A., Doris, L., Sandra, B., Dilip, G., Elizabeth, M., Morris, E.A. and Katja, P. (2019) Quantitative in Vivo Proton MR Spectroscopic Assessment of Lipid Metabolism: Value for Breast Cancer Diagnosis and Prognosis. Journal of Magnetic Resonance Imaging, 50, 239-249. https://doi.org/10.1002/jmri.26622

[23] Galati, F., Luciani, M.L., Caramanico, C., Moffa, G., Catalano, C. and Pediconi, F. (2019) Breast Magnetic Resonance Spectroscopy at $3 \mathrm{~T}$ in Biopsy-Proven Breast Cancers: Does Choline Peak Correlate with Prognostic Factors. Investigative Radiology, 54, 767-773. https://doi.org/10.1097/RLI.0000000000000597

[24] Vassiou, K., Tsougos, I., Kousi, E., Vlychou, M., Athanasiou, E., Theodorou, K., Arvanitis, D.L. and Fezoulidis, I.V. (2013) Application Value of 3T ${ }^{1} \mathrm{H}$-Magnetic Resonance Spectroscopy in Diagnosing Breast Tumors. Acta Radiologica (Stockholm, Sweden: 1987), 54, 380-388. https://doi.org/10.1177/0284185113475921

[25] Jena, A., Taneja, S. and Mehta, S.B. (2012) Integrated Quantitative DCE-MRI and DW-MRI to Characterize Breast Lesions. European Journal of Radiology, 81, S64-S65. https://doi.org/10.1016/S0720-048X(12)70025-6

[26] Wan, W., Chen, S.L., Tian, T.Q., Chen, J.L., Xu, X.L. and Liu, W. (2012) The Differential Value of Breast MRT1 WI Dynamic Enhancement Combined with T2 WI First Perfusion Imaging for Benign and Malignant Breast Lesions. Journal of Clinical Radiology Journal of Learning, 31, 184-187.

[27] Yuan, L. and Zhu, B. (2008) Clinical Application Value of MRI Perfusion Imaging in Breast Tumors. Medical Journal, No. 7, 775-777.

[28] Li, C., Meng, S., Yang, X., et al. (2014) The Value of T2 in Differentiating Metastatic from Benign Axillary Lymph Nodes in Patients with Breast Cancer: A Preliminary in Vivo Study. PLoS ONE, 9, e84038. https://doi.org/10.1371/journal.pone.0084038

[29] Wang, X.H., Peng, W.J., Tang, F., et al. (2010) Application of T2 Magnetic Resonance Perfusion Imaging in Monitoring the Efficacy of Neoadjuvant Chemotherapy for Breast Cancer. Journal of Practical Radiology, 26, 1323-1327.

[30] Plecha, D.M. and Faulhaber, P. (2017) PET/MRI in Breast Cancer. European Journal of Radiology, 94, A26-A34. https://doi.org/10.1016/j.ejrad.2017.05.006

[31] Moy, L., Noz, M.E., Maguire, J., et al. (2010) Role of Fusion of Prone FDG-PET and Magnetic Resonance Imaging of the Breasts in the Evaluation of Breast Cancer. The Breast Journal, 16, 369-376. https://doi.org/10.1111/j.1524-4741.2010.00927.x

[32] Xu, Y.F., Liang, J.T., Wang, F.X., et al. (2019) Preliminary Comparative Study of 18F-FDG PET/MR and PET/CT in the Diagnosis of Breast Tumors. Journal of Clinical Radiology, 38, 815-820.

[33] Gillies, R.J., Anderson, A.R., Gatenby, R.A., et al. (2010) The Biology Underlying Molecular Imaging in Oncology: From Genome to Anatome and Back Again. Clin- 
ical Radiology, 65, 517-521. https://doi.org/10.1016/j.crad.2010.04.005

[34] Lambin, P., Rios-Velazquez, E., Leijenaar, R., et al. (2012) Radiomics: Extracting More Information from Medical Images Using Advanced Feature Analysis. European Journal of Cancer, 48, 441. https://doi.org/10.1016/j.ejca.2011.11.036

[35] Levy, M.A., Freymann, J.B., Kirby, J.S., et al. (2012) Informatics Methods to Enable Sharing of Quantitative Imaging Research Data. Magnetic Resonance Imaging, 30, 1249-1256. https://doi.org/10.1016/j.mri.2012.04.007

[36] Parekh, V. and Jacobs, M.A. (2016) Radiomics: A New Application from Established Techniques. Expert Review of Precision Medicine and Drug Development, 1, 207-226. https://doi.org/10.1080/23808993.2016.1164013

[37] Whitney, H.M., Taylor, N.S., Drukker, K., et al. (2019) Additive Benefit of Radiomics over Size Alone in the Distinction between Benign Lesions and Luminal a Cancers on a Large Clinical Breast MRI Dataset. Academic Radiology, 26, 202-209. https://doi.org/10.1016/j.acra.2018.04.019

[38] Keller, P.J., Lin, A.F., Arendt, L.M., et al. (2010) Mapping the Cellular and Molecular Heterogeneity of Normal and Malignant Breast Tissues and Cultured Cell Lines. Breast Cancer Research, 12, R87. https://doi.org/10.1186/bcr2755

[39] Hui, L., Zhu, Y., Burnside, E.S., et al. (2016) MR Imaging Radiomics Signatures for Predicting the Risk of Breast Cancer Recurrence as Given by Research Versions of Mamma Print, Oncotype DX, and PAM50 Gene Assays. Radiology, 281, 382-391. https://doi.org/10.1148/radiol.2016152110

[40] Sala, E., Mema, E., Himoto, Y., et al. (2017) Unravelling Tumour Heterogeneity Using Next-Generation Imaging: Radiomics, Radiogenomics, and Habitat Imaging. Clinical Radiology, 72, 3-10. https://doi.org/10.1016/j.crad.2016.09.013

[41] Zhao, G.Y., Yin, J.D., Guo, W.L., et al. (2019) Investigation and Analysis of the Use of Medical Magnetic Resonance Equipment in 2018. China Medical Equipment, 34, $15-18$.

[42] Qiu, X.L., Yao, S.M. and Bao, J.L. (2018) Application Performance Evaluation of Domestic Superconducting Magnetic Resonance Imaging Equipment. China Medical Equipment, 33, 65-69.

[43] Wang, L.J., Bi, F., Zhang, L.F., et al. (2015) Investigation and Analysis of Maturity of Domestic Medical Equipment. China Medical Equipment, 30, 6-9. 